\title{
Combining Research With "Servicing" to Enhance Sport Performance
}

In high-performance environments, the interaction of sport scientists with coaches and athletes is often referred to as servicing. In some cases, providing services to athletes (eg, collecting training data) is considered one of the primary roles of the sport scientist. In both professional and Olympic sports, research is rarely considered a priority, often thought to have little value and, for some, an activity that only "academics" embrace. However, as the assessment of the physiological, psychological, and skill-based determinants of elite athlete performance continues to progress in complexity and sophistication, it may be the case that athlete servicing and research should be viewed on a continuum rather than as 2 separate entities.

As is to be expected, winning is the primary objective for highperformance teams. Thus, coaches and their support staff are often interested in understanding demands of competition, fitness characteristics of successful athletes, limitations to performance, and training methods that can improve an athlete's performance. It is common for sport scientists working in high-performance settings to develop real-world questions based on interpretation of their data, collaborate with coaches to design and implement interventions derived from these ideas, conduct further testing after the interventions, and use the outcomes of this testing to reevaluate the hypotheses, with this entire process repeated cyclically. Implicitly, then, sport scientists are following the scientific method and performing research, even though this is not widely recognized in the sport industry.

One important aspect of the evolution of monitoring elite athletes is the transition of athlete testing from the laboratory to the field. Laboratory testing is often considered to have high reliability but low ecological validity, while field assessments may have lower reliability but strong ecological validity. With the advent of wearable technologies, markerless motion-analysis systems, and sophisticated competition-analysis tools, there has been a rapid expansion of the ability to obtain high-quality data in field situations. Athlete testing can therefore be conducted in the context of routine training sessions and competitions, enabling much more frequent athlete monitoring. Laboratory tests performed several weeks or months apart yield only a series of "snapshots" of the measured variables. By contrast, field testing can be conducted daily, permitting much greater resolution in the detection of trends and easier identification of abnormal results. Even where data collected in training and competition environments are slightly less controllable than those obtained in the laboratory (and confounding factors), the ability to collect data much more frequently may lead to more useful conclusions, especially if sufficient contextual information is also collected.

With the rise of noninvasive athlete-measurement tools, there has been an abundance of new information, and each training session can be seen as a test that can provide valuable information. Important steps need to be taken in the collection, storage, and treatment of training and competition data. In particular, it is essential that information collected from the field be valid and reliable and that good data-management systems and gold-standard research-quality data-curation procedures (ie, naming conventions, data integration, security, etc) be followed. Data collected by scientists in the field are important to the wider sport-science community, particularly as they are often derived from case studies on truly exceptional athletes.

Note, however, that despite the relative ease of data collection in training and competition, it is essential that scientists remain focused on quality assurance of the data obtained. High tech does not always result in high-quality data. Surprisingly few of the emerging micro and digital technologies have been subject to independent validation or assessment of measurement accuracy. The same can be said for the numerous brief psychometric questionnaires that are frequently used in the field. Accordingly, with the shift of sport scientists out of the laboratory and into the field, it is important that they select appropriate measurement tools, construct integrated monitoring systems, and develop datamanagement skills to leverage these emerging opportunities. It is also essential that fundamental coaching and soft skills be retained as the translation of new insights obtained from athlete monitoring into effective behavior changes (for athletes and coaches) remains a critical skill for sport scientists.

With the high availability of data, and application of the scientific process, scientists are often motivated to publish their findings in journals such as IJSPP. While there is some concern over the potential loss of intellectual property from using training and competition data in publications, the peer-review process will ultimately improve our understanding of professional practice. In addition, there are concerns over data ownership, and caution is required to ensure that all stakeholders have provided permission for use of data for public release and/or research purposes. Accordingly, it is essential that athletes, coaches, and stakeholder organizations provide informed consent and that appropriate ethical approval is sought before planning to publish these data.

Publication of the findings, however, is not always a simple process, since ownership of the data often falls to the organizations employing the sport scientists, and these organizations can be quite zealous in seeking to protect their intellectual property. Resistance to the notion of sharing "trade secrets" and inside information with potential competitors is common, and this can make it difficult for sport scientists to gain official or even tacit approval to publish. However, research conducted on a specific population is not always readily transferable to other groups or individuals. Differences in subject populations, physical and cultural environments, access to equipment and facilities, and varying methods of intervention tend to inhibit direct translation of athlete- or team-derived knowledge and information. Publishing data collected in the training environment also ensures that methods used are exposed to the peer-review process and enhances accountability of the individuals collecting the data. Feedback from peers can lead to insights that would never have emerged if the data were kept entirely in-house. Although all these points may be valid, the argument for publishing can still be difficult to win. 
With an increase in research-quality data collection in the field, improvements in sport-specific tests, and the benefits of ecological research, the distinction between data collected from "servicing" and laboratory-based research is now blurred. The collection of data in the daily training environment in a controlled and precise manner allows the accurate determination of change in meaningful variables on both a group and an individual level and ultimately enhances our ability to prescribe training and minimize risk of underperformance, fatigue, and injury. Furthermore, this approach may enhance the perceived value and professionalism of sport science. The convergence of servicing and research is likely to continue and become more pronounced as the pace of the technological revolution becomes even greater, with sport science a major beneficiary.

Shona L. Halson, Australian Catholic University, IJSPP Associate Editor

Alan G. Hahn, Queensland Academy of Sport, University of Canberra Research Institute for Sport \& Exercise, Griffith University School of Engineering

Aaron J. Coutts, University of Technology Sydney 